\title{
In silico and microbiological studies for reduction of Pseudomonas aeruginosa virulence factors controlled by quorum sensing.
}

\author{
Amira Etman*1, Heba M. Abostate, Dalia A. El-Damasy, Dalia H. Soliman ${ }^{2,3}$ and Sahar M R. \\ Radwan', ${ }^{1,4}$ \\ ${ }^{1}$ Microbiology \& Immunology department, Faculty of pharmacy, Egyptian Russian University. \\ ${ }^{2}$ Pharmaceutical chemistry department, Faculty of pharmacy, Egyptian Russian University. \\ ${ }^{3}$ Pharmaceutical chemistry department, Faculty of Pharmacy (Girls), Al-Azhar University. \\ ${ }^{4}$ Microbiology \& Immunology department, Faculty of Pharmacy (Girls), Al-Azhar University. \\ * Correspondence: e-mail amira.gamal.eru@gmail.com ; Tel.: +2 01094025591
}

Article history: Received 09-12-2020 Revised 21-12-2020 Accepted 07-01-2021

\begin{abstract}
Introduction: $P$. aeruginosa is one of the major causes of. $P$. aeruginosa's virulence factors are highly controlled by QS systems which lead to antibiotics resistance. Targeting QS may be a promising therapeutic hope for $P$. aeruginosa infections. Aim of work: Inhibition of some quorum sensing (QS) regulated virulence factors via using non-antibiotic helping drugs to overcome antibiotic resistance problem. Methods: Molecular Docking was performed using the required constraints in the binding sites of the Las \& $\mathrm{Rhl}$ receptor proteins of $P$. aeruginosa to estimate the expected activity of (quercetin and meloxicam) and compare them with great docking scores compounds like rosmarinic acid. PAO1 was used as reference strain with other $P$. aeruginosa strains (clinical samples). An antibiotic sensitivity test was performed to evaluate antibiotic resistance. The minimum inhibitory concentration (MIC) of tested drugs were also measured against the resistant strains. Virulence factors thanked for QS (protease, pyocyanine, rhamnolipids) and biofilm formation of selected strains were assessed before and after treatment with quercetin \& meloxicam. Results and conclusion: In silico studies indicated that quercetin and meloxicam could act as inhibitors for the auto-inducer molecules as they have a high affinity for the regulatory proteins of the QS system lasR and rhIR. The experimental study of quercetin and meloxicam against $P$. aeruginosa showed that at sub-MIC, both drugs reduced biofilm formation and some tested virulence factors. Treatment of $P$. aeruginosa infection using QS inhibitors is expected and this approach may overcome the antibiotic resistance problem.
\end{abstract}

Keywords: Quorum sensing; P. aeruginosa; Virulence factors; Quercetin; Meloxicam.

\section{INTRODUCTION}

P. aeruginosa is a vital nosocomial health care associated pathogen to blame for a range of infections with limited therapeutic options thanks to its antibiotic resistance ${ }^{1}$. Production of many virulence factors (protease, pyocyanin, rhamnolipids) and biofilm formation let this pathogen become a successful agent ${ }^{2}$. This bacterium survives in two forms: the planktonic form and in surface-conducted communities named biofilms. These biofilm structures possess three-dimensional shapes, below the biofilm

umbrella cells connected chemically and protective castles were built to ensure their survival from natural host defenses and antibiotics. Many studies are made to spot novel drugs and targets trying to resolve this problem ${ }^{3,4}$. Pyocyanin is a chemical compound (phenazine) that makes reactive oxygen intermediates $^{5}$. Pyocyanin causes depleting intracellular c-AMP and ATP levels ${ }^{6,7}$. Additionally, it considers as a host immune modifier that enhances system evasion during chronic infection evolution? Proteases are hydrolyzing enzymes that target host cell proteins and interfere with their system because of the proliferation ability of bacteria through the infected host ${ }^{8,9}$.

Cite this article : Etman, A., Abostate, H., El-Damasy, D., Soliman, D., Radwan, S. In silico and Microbiological studies for reduction of Pseudomonas aeruginosa virulence factors controlled by quorum sensing. Azhar International Journal of Pharmaceutical and Medical Sciences, 2021; 1(1): 40-53. 
Rhamnolipids (RLs), glycolipid biosurfactants, are thanked for the deterioration of patients having ventilator-associated pneumonia ${ }^{10}$. Rhamnolipids solubilize lung surfactant, enabling phospholipase $\mathrm{C}$ entry within cell membranes, and that (RLs) play a critical role in managing biofilm formation ${ }^{11}$. Furthermore, rhamnolipids incorporate into vegetative cell membranes and interrupt tight junctions ${ }^{12,13}$. Also, rhamnolipids can lead to polymorphonuclear (PMN) leukocytes, macrophages death and suppress phagocytosis ${ }^{14}$.

This pathogenic bacterium is thought to use bacterial connection system that depends on cell concentration called quorum sensing (QS) to manage a lot of virulence factors, related to its pathogenicity. Bacterial Quorum sensing means the ability of a bacterium to receive information from other members within the population upon reaching a concentration ${ }^{15}$. The quorum sensing fact, or concentration-dependent communication, starts when one bacterium releases autoinducers (AIs) into the surrounding environment, their concentration is simply too low to be sensed. However, when the bacterial concentration increase, the strength of AI concentrations enables the bacteria, to regulate target genes $^{16}$. P. aeruginosa possesses two major QS systems, last/lasR and rhlI/rhlR.

\section{3-oxododecanoyl-Lhomoserine}

lactone (3-oxo-C12-AHL) and N-butanoyl homoserine lactone (C4-HSL) are the autoinducers that cognate the transcriptional regulators, LasR and RhlR respectively, and synthesized by the synthase genes. The LasR/3OC12-HSL and RhlR/ C4-HSL complexes, modulate the expression of genes responsible to produce virulence factors ${ }^{15}$. Due to this bacterial gift, the common antibiotic treatments are often not effective against $P$. aeruginosa persistent infections. Colistin, a polymyxin antibiotic, is considered since the use of pis aller therapy for multi-drug resistant (MDR) $P$. aeruginosa is limited due to its extreme cytotoxicity. The generation of colistin-resistant strains is recorded from different areas worldwide warranting serious measures ${ }^{17,18}$. The use of quorum sensing inhibitors increases the sensitivity of existing antibiotics. Contrary to traditional antibiotics which target the essential cellular vital processes, the quorum sensing inhibitors (QSIs) are considered to inhibit the bacterial connection without exerting selective pressure on the bacteria, so decreasing the possibility for the looks of MDR strains. Some natural and artificial compounds are investigated for their ability to stop the bacterial QS circuit and attenuate pathogenicity, apart from any harmful side-effect ${ }^{19}$.
Computer-aided study of molecular docking is now commonly recognized for finding new hits for targets of known protein structures ${ }^{20}$. It may be a promising area of research to identify novel drugs with quorum sensing inhibitory (QSI) potential through Computer-Aided Drug Discovery (CADD). Therefore, we aimed to inhibit quorum sensing related virulence factors by using natural compounds or purpose redirecting of already existing drugs hoping to unravel the antibiotic resistance problem in treating $P$. aeruginosa infection ${ }^{21}$.

In the present study, a docking study was conducted to predict whether the compounds would have a comparable binding mode to the rosmarinic acid and therefore the co-crystallized compound within the LasR and RhlR receptor binding sites. It also offers some insight into the binding affinity of the compounds into LasR and RhlR receptor proteins supported the docking scores, binding modes, and field alignments of meloxicam moreover as quercetin, they were selected to estimate their QSI efficiency using in vitro experimental methods.

\section{MATERIALS AND METHODS}

\subsection{Docking studies}

Automated docking studies were meted out using Glide (SP) scoring function Maestro10.1 Schrodinger, (2015-Release-4). Flexible docking was performed to all ligands to LasR and RhlR receptor proteins. The LasR protein (ID: 2UV0) structure was downloaded from http://www.pdb.org. The designed RhlR protein (ID: P54292.1) was obtained from the SWISS-MODEL: Automated protein homology-modeling server http://swissmodel.expasy.org/.

\subsubsection{Ligand Preparation}

LigPrep module was used for the geometrical refining of chemical structures. LigPrep is designed to match premium 3D structures with correct chiralities. Ionization original states have been retained; tautomers and conformations have been developed by the city method as implemented in MacroModel version 9.8, 2010, using the force field OPLS-2005. Subsequently, the produced conformers were minimized using a truncated Newton conjugate gradient (TNCG) minimization of up to 500 iterations. The $30 \mathrm{kcal} / \mathrm{mol}$ energy difference conformers as compared to the worldwide energy minimum conformer were retained. The conformational searches were dispensed for a solution using the generalized born/solvent 
accessible surface (GB/SA) continuum solvation model.

\subsubsection{Protein Preparation}

For protein preparation, wizard Maestro software was used. The selected chains were manipulated for missing hydrogens and for assigning proper bond orders. The H-bonds were optimized using sample orientation. All the polar hydrogens have been shown. All crystallographic waters without chemical bond interactions with protein residues have been excluded. Finally, the protein structure of non-hydrogen atoms has been reduced to the default Root Mean Square Deviation (RMSD) of 0.3 .

\subsubsection{Receptor Grid Generation}

From the pinpointed receptor, the co-crystalline ligand was isolated from the receptor chain site. The atoms were 1.0> Van der Waals radii capable of scale, while the partial atomic charge was 0.25 by nature. The location represents the enclosure box at the middle of the workspace ligand. Following this protocol, a ligand-centered grid was created using the default Glide settings. Both ligands have been docked into this grid structure.

\subsubsection{Molecular Docking Analysis}

Flexible docking on the specified receptor grid was performed using the Glide Module Precision (SP) feature, version 5.6, 2010. Constraints to pinpointed ligand-receptor interactions have not been set. The output format of the structure was set to show the viewer file so that the output of the resulting docking studies could be displayed from the pose viewer.

\subsection{Field alignment study}

Surface Alignment Analysis enables the alignment of any molecule drawn in $2 \mathrm{D}$ with the current 3D prototype and the estimation of the field similarity value. In this way compare other actives or unknowns to active lead compounds and see how the trend of the sector shifts and which field points are maintained in the actives. It is not intended to direct a logical approach focused on the discovery and exploitation of molecular field similarities. The sector alignment technique offered by Cresset's FieldAlign Module, version 2.0.1, was not intended to align the candidates with the bioactive lead compound $\mathrm{OHN}$.

\subsection{Bacterial strain and growth condition}

P. aeruginosa PAO1gifted by Dr. Dalia El Damasy from Zagazig University was used as reference strain altogether in the experiments. $P$. aeruginosa isolates were previously isolated from several clinical samples and identified using Micro Scan. All bacterial cultures were maintained in TSB broth at $37^{\circ} \mathrm{C}$.

\subsection{Minimal inhibitory concentration (MIC)}

Broth microdilution method, quercetin was first dissolved in DMSO and then diluted in MullerHinton broth at the necessary final concentration. Two-fold dilutions were prepared in a very 96-well plate with a final quercetin concentration ranging from 1000 to $11,7 \mu \mathrm{g} / \mathrm{ml}$ and meloxicam at concentrations ranging from 1,000 to $1,95 \mu \mathrm{g} / \mathrm{ml}$. Bacterial suspensions with incipient turbidity of 0.05 (OD600) were used. The bacterial suspension (100 $\mu \mathrm{l})$ was inoculated and the microtiter plates were incubated at $37^{\circ} \mathrm{C}$ for about 16 to 20 hours.

\subsection{Antibiotic sensitivity test}

\subsubsection{Microscan Negative Breakpoint Combo Panel type 44 (NBC 44) (DADE BEHRING, West Sacramento, USA)}

Microscan negative breakpoint combo panel type 44 is an in vitro diagnostic product for testing antimicrobial susceptibility of Gram-negative bacteria based upon many antimicrobial agent susceptibility tests dosed and dried into the Microscan panel and for automated identification of Gram-negative facultative bacilli to species level.

\subsection{Detection of QS-related virulence factors}

\subsubsection{Assessment of biofilm with the crystal violet method}

The two drugs tested for impact on biofilm formation were performed as defined by Stepanovic et al. (2007) ${ }^{22}$ with minor modifications. Shortly, PAO1 and clinical isolate cells were cultured overnight in the LB medium then they were diluted 1:100 in the fresh LB medium for biofilm processing. PAO1 and one of each clinical isolate were cultured with each drug at different sub-inhibiting concentrations of quercetin (187.5 to $11.7 \mu \mathrm{g} / \mathrm{ml}$ ) and meloxicam (15.62 to $1.9 \mu \mathrm{g} / \mathrm{ml}$ ) for 24 hours at $37^{\circ} \mathrm{C}$. Subsequently, non-adherent bacteria were eliminated by washing with sterile phosphate-buffered saline (PBS) and the adhering bacteria were stained with a $1 \%$ crystal violet 
solution for $10 \mathrm{~min}$. PBS cleaned the wells to remove excess stain. After air-dried wells, the purple crystal bound to the biofilm was extracted with $30 \%$ acetic acid. The absorbance of the stained biofilm was measured spectrophotometrically at OD590. And the percentage of inhibition was determined following the following equation

$\%$ inhibition $=($ Control OD - Test OD $) /$ Control OD $\times 100^{23}$.

\subsubsection{Skimmed milk assessment for protease activity of pseudomonas}

PAO1 and clinical isolates were overnight cultured with those two drugs at sub-inhibitory concentrations quercetin $(93.75 \mu \mathrm{g} / \mathrm{ml})$, meloxicam $(15.62 \mu \mathrm{g} / \mathrm{ml})$ and a negative control (phosphate buffer saline). Centrifugation for $15 \mathrm{~min}$ at 10,000 rpm, then supernatants collection and were aliquoted into the cups made in skimmed milk agar plates. Incubation at $37^{\circ} \mathrm{C}$ for twenty-four $\mathrm{h}$ took place. The clearance zone diameter around each well was measured, which were resembling protease activity 24 .

\subsubsection{Rhamnolipid screening before and after treatment}

\subsubsection{Oil spreading method}

The system for the spread of oil was administered according to the tactics mentioned in ${ }^{25}$, ${ }^{26}$. In short $5 \mathrm{ml}$ of water was applied to the Petri dishes $(15 \mathrm{~cm}$ in diameter) after the addition of $10 \mathrm{ml}$ of oil-stained with (Sudan black or Eosin) adjustment by (Shamaa et al., 2019) ${ }^{27}$ to the surface of the water. Subsequently, $1 \mathrm{ml}$ of culture supernatant was lowered to the oil surface. The clear zone diameter formed on the oil surface was compared to $10 \mathrm{ml} \mathrm{H}_{2} \mathrm{O}$ as a negative regulation.

\subsubsection{Emulsification Measurement}

The emulsification operation was calculated by Cooper et al technique, $1987^{28}$ with a slight adjustment. To $2 \mathrm{ml}$ of supernatant culture, $2 \mathrm{ml}$ of sunflower seed oil was applied and vortexed at high speed for two minutes. The mixture was permitted to square for 10 minutes before the measurement.

The emulsification activity is defined as the height of the emulsion layer divided by the total height and represented as a percentage of ${ }^{29}$.

\subsubsection{Pyocyanin production measurement}

The development of pyocyanine was calculated by the extraction of chloroform- $\mathrm{HCl}$ as defined by Essar and Associates (1990) 30 with some modifications. In short, $7.5 \mathrm{ml}$ of PAO1 supernatant and clinical isolate cultures treated with or without 2 drugs were first extracted at a sub-inhibiting concentration of quercetin $(62.5 \mu \mathrm{g} / \mathrm{ml})$ and meloxicam $(16 \mu \mathrm{g} / \mathrm{ml})$ with $4.5 \mathrm{ml}$ of chloroform followed by $1 \mathrm{ml}$ of $\mathrm{HCl}$ re-extraction $(0.2 \mathrm{M})$. The response absorbance was measured at $520 \mathrm{~nm}$. The concentration of pyocyanin was determined as follows:

Pyocyanine concentration $=$ OD520 $\times 17.072$.

\subsection{Statistical Analysis}

Microsoft Excel 2013 (Redmond, WA, USA) was used for statistical analysis of data of 95 percent significance. One-way variance analysis (ANOVA) was performed to determine statistical differences between extractions using Tuckey post-hockey test and IBM SPSS statistics 22 software (Armonk, NY, USA).

\section{RESULTS}

\subsection{In silico study results}

The results of the docking scores, represented in Table 1, of rosmarinic acid and quercetin into the active site of the target protein LasR showed a significant difference between them to the side of the tested drugs, additionally, the binding mode and interactions were better too. As for the target RhlR Table 2 docking scores of rosmarinic acid, quercetin and meloxicam were close, with the quercetin showing a better binding mode inside the pocket. The overlay of the three compounds into the active site of $P$. aeruginosa RhlR (ID: $2 \mathrm{q} 0 \mathrm{o}$ ) showed an interaction pattern in the binding site that maintained the hydrogen bond interaction with the active site residues ASP72, THR131, HIS53, MET129, and TRP59, (Figure 1). These same target compounds showed good binding affinities into the active site of $P$. aeruginosa LasR (ID: 2 uvo) as well as hydrogen bond interactions with the key residues, ASP73, LEU 125, TYR 56, SER129, THR115, and ARG 61, (Figure 2). 
Table 1: The docking results of the tested compounds into the active sites of LasR (ID: $2 \mathrm{uv} 0$ ):

\begin{tabular}{|c|c|c|c|}
\hline Compound & $\begin{array}{ll}\text { Docking } & \text { score } \\
(\mathrm{Kcal} / \mathrm{mol}) & \\
\end{array}$ & Interacting residues & distance \\
\hline Rosmarinic acid & -9.78 & $\begin{array}{l}\text { ASP } 73 \text { (E) H-donor } \\
\text { LEU } 125 \text { (E) H-donor } \\
\text { TYR } 56 \text { (E) H-acceptor } \\
\text { SER } 129 \text { (E) H-acceptor }\end{array}$ & $\begin{array}{c}2.93 \\
2.94 \\
2.11 \\
2.85\end{array}$ \\
\hline Quercetin & -7.28 & $\begin{array}{lll}\text { THR } & 115 & \text { H-donor } \\
\text { ASP } & 73 & \text { H-donor } \\
\text { TRP } & 60 & \text { H-acceptor } \\
\text { ARG } & 61 & \text { H-acceptor } \\
\text { ARG } & 61 & \text { H-acceptor } \\
\text { ARG } & 61 & \text { H-acceptor } \\
\text { ARG } & 61 & \text { ionic } \\
\text { ARG } & 61 & \text { ionic }\end{array}$ & $\begin{array}{l}2.87 \\
3.28 \\
2.90 \\
3.40 \\
3.32 \\
2.63 \\
3.82 \\
2.63\end{array}$ \\
\hline Meloxicam & -7.73 & $\begin{array}{lll}\text { ARG } & 61 & \text { H-acceptor } \\
\text { SER } & 129 & \text { H-acceptor } \\
\text { TRP } & 60 & \text { H-acceptor } \\
\text { ALA } & 127 & \text { H-acceptor } \\
\text { ARG } & 61 & \text { ionic } \\
\text { ARG } & 61 & \\
\end{array}$ & $\begin{array}{c}3.15 \\
2.88 \\
2.97 \\
3.22 \\
3.91 \\
3.15\end{array}$ \\
\hline
\end{tabular}

Table 2: The docking results of the tested compounds into the active sites of RhlR (ID: $2 q 0 o$ ):

\begin{tabular}{|c|c|c|c|}
\hline Compound & $\begin{array}{l}\text { Docking } \\
\text { score } \\
\text { (Kcal/mol) }\end{array}$ & $\begin{array}{l}\text { Interacting } \\
\text { residues }\end{array}$ & distance \\
\hline \multirow{5}{*}{ Rosmarinic acids } & \multirow{5}{*}{-6.73} & ASP 72 (A) & \multirow{5}{*}{$\begin{array}{l}2.98 \\
2.90\end{array}$} \\
\hline & & H-donor & \\
\hline & & THR 131 & \\
\hline & & (A) & \\
\hline & & H-acceptor & \\
\hline \multirow{7}{*}{ Quercetin } & \multirow{7}{*}{-6.75} & ASP 72 (A) & \\
\hline & & H-dnor & \\
\hline & & THR 131 & 2.91 \\
\hline & & (A) & 3.48 \\
\hline & & $\mathrm{H}$-acceptor & 3.74 \\
\hline & & HIS 53 (A) & \\
\hline & & H-pi & \\
\hline \multirow{9}{*}{ Meloxicam } & \multirow{9}{*}{-6.53} & MET 129 & \\
\hline & & (A) & \\
\hline & & H-donor & \\
\hline & & $\begin{array}{ll}\text { TRP } & 59\end{array}$ & 4.32 \\
\hline & & (A) & 3.04 \\
\hline & & H-acceptor & 3.38 \\
\hline & & THR & \\
\hline & & $131(\mathrm{~A})$ & \\
\hline & & H-acceptor & \\
\hline
\end{tabular}




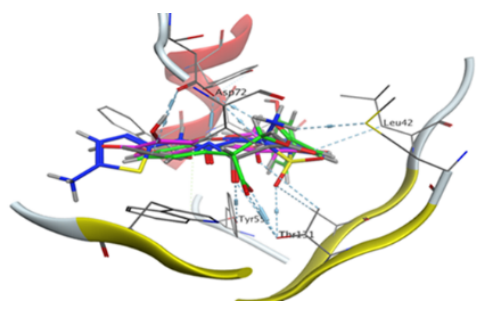

Figure (1): The 3D representation of the overlay of meloxicam (blue), rosmarinic acid (green), quercetin (purple) with the N-3-oxo-dodecanoyl-L-homoserine lactone $(\mathrm{OHN})$ and their interactions with the key residues into the binding site of $P$. aeruginosa RhlR (ID: 2q0o)

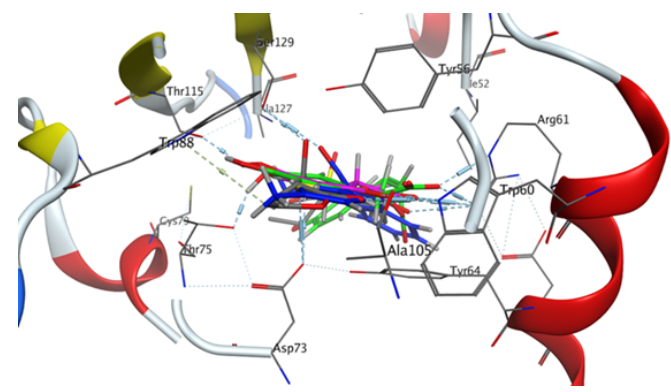

Figure (2): The 3D representation of the overlay of meloxicam (blue), rosmarinic acid (green), quercetin (purple) with the N-3-oxo-dodecanoyl-L-homoserine lactone and their interactions with the key residues into the binding site of $P$. aeruginosa LasR (ID: 2uvo)

Alignment field studies also showed the great similarity of the molecular fields that are demonstrated by the good alignment scores, of both of them to the original ligand and upon applying rosmarinic acid as the reference ligand quercetin also demonstrated very good alignment to this reference, Figures 3-8. Both molecular docking analysis and alignment field study revealed that our tested drugs showed a good binding affinity with the active sites of $P$. aeruginosa LasR \& RhlR and also a great similarity between them with the original ligand and also with the reference compound rosmarinic acid. This prompted us to examine the quorum sensing inhibitory (QSI) potential effect of the tested compounds with more hope to develop potential QSIs from natural resources also through purpose redirecting such as testing meloxicam

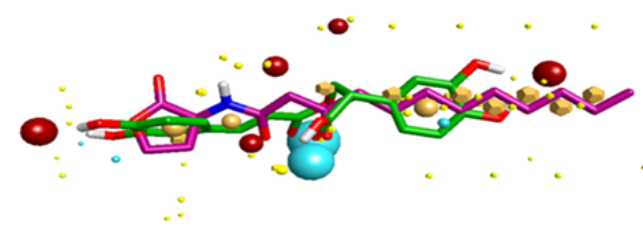

Figure (3): Field alignment of romarinic acid (green) and OHN (magenta) with alignment score 0.558 , showing similar molecular fields, suggesting a similar binding mode. Cyan: negative field points, Red: positive field points, Yellow: van der Waals surface field points, Gold: hydrophobic field points.

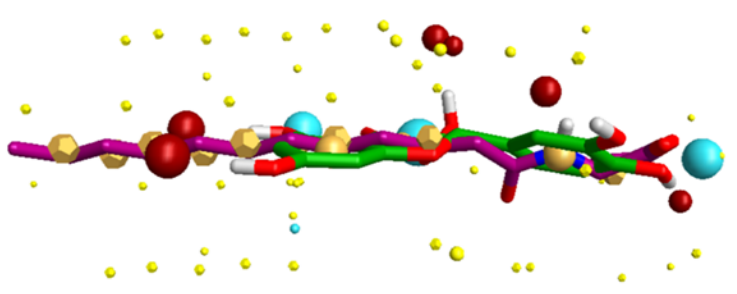

Figure (4): Field alignment of quercetin (green) and $\mathrm{OHN}$ (magenta) with alignment score 0.514 , showing similar molecular fields, suggesting a similar binding mode. Cyan: negative field points, Red: positive field points, Yellow: van der Waals surface field points, Gold: hydrophobic field points.

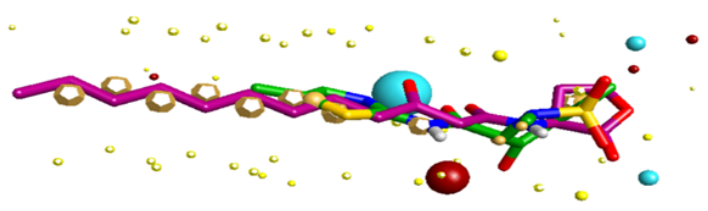

Figure (5): Field alignment of meloxicam (green) and OHN (magenta) with alignment score 0.61 , showing similar molecular fields, suggesting a similar binding mode. Cyan: negative field points, Red: positive field points, Yellow: van der Waals surface field points, Gold: hydrophobic field points. 


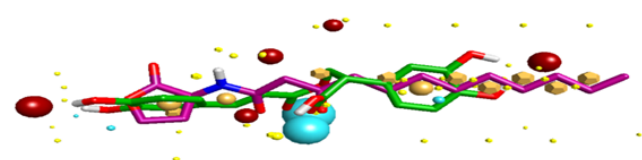

Figure (6): Field alignment of romarinic acid (green) and $\mathrm{OHN}$ (magenta) with alignment score 0.558 , showing similar molecular fields, suggesting a similar binding mode. Cyan: negative field points, Red: positive field points, Yellow: van der Waals surface field points, Gold: hydrophobic field points.

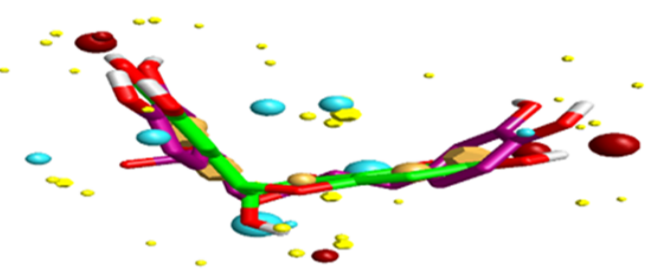

Figure (7): Field alignment of quercetin (green) and romarinic acid (magenta) with alignment score 0.687 , showing similar molecular fields, suggesting a similar binding mode. Cyan: negative field points, Red: positive field points, Yellow: van der Waals surface field points, Gold: hydrophobic field points.

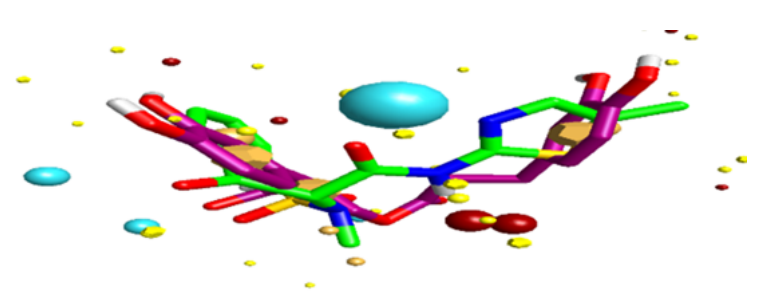

Figure (8): Field alignment of miloxicam (green) and romarinic acid (magenta) with alignment score 0.55 , showing similar molecular fields, suggesting a similar binding mode. Cyan: negative field points, Red: positive field points, Yellow: van der Waals surface field points, Gold: hydrophobic field points

\subsection{Experimental study}

\subsubsection{Bacterial strains used}

In this study, 20 bacterial strains were previously isolated from different clinical samples and identified as $P$. aeruginosa. After performing antibiotic sensitivity test using MICRO-SCAN, six $P$. aeruginosa strains named as XDR (Extensively drug-resistant) as they showed high resistance to many important antibiotics like Levofloxacin, Cefriaxon, Tobramycin, Cifibime, Tinam, and Amikin (Figure 9) only one will be used in this study as an example of clinical isolates and named as P1.

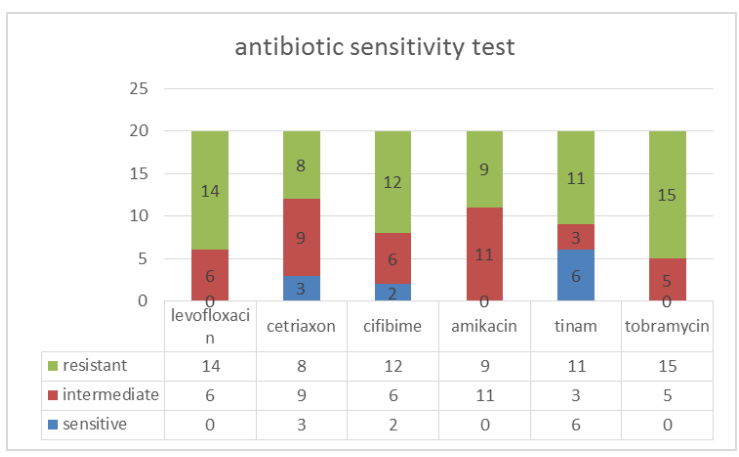

Figure (9): The effect of different antibiotics on

Pseudomonas aeruginosa clinical isolates.

\subsubsection{Minimal inhibitory concentration (MIC) results}

The MICs of the two drugs against PAO1 and P1 were measured via the broth microdilution method according to the standard Clinical and Laboratory Standards Institute. The MIC of quercetin was $\geq 375 \mu \mathrm{g} / \mathrm{ml}$ and meloxicam was $\geq$ $31.25 \mu \mathrm{g} / \mathrm{ml}$.

\subsubsection{The Crystal violet method for biofilm assessment}

Regarding the OD readings of the crystal violet method showed that quercetin caused $60 \%$ reduction in biofilm formation at $93.75 \mu \mathrm{g} / \mathrm{ml}$ with PAO1 and converting it from strong to moderate biofilm forming. While meloxicam caused $56 \%$ inhibition at $15.62 \mu \mathrm{g} / \mathrm{ml}$ with PAO1 and it converts from strong to moderate biofilm-forming (The mean difference is significant at the 0.05 level). (Figure 10) 


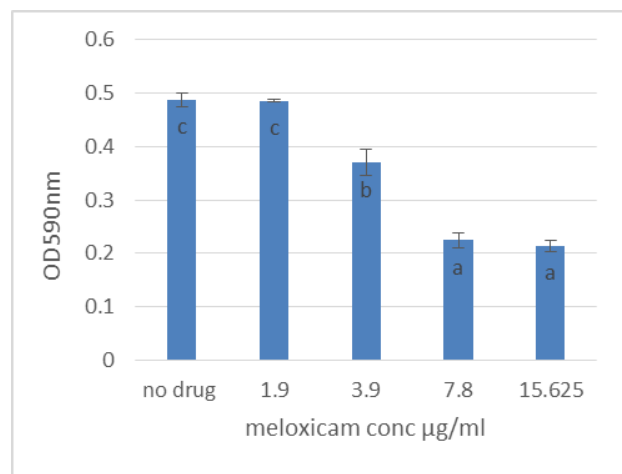

(a)

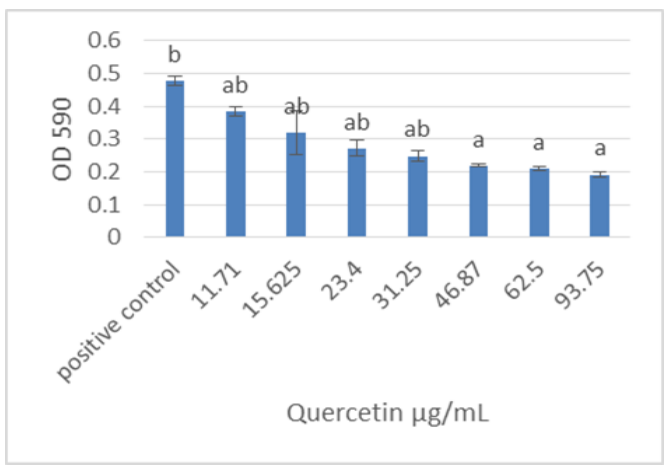

(b)

Figure (10): (a) OD readings at 590nm of bioflim formation ability by crystal violet methods using quercetin at sub-inhibitory conc as an anti-biofilm with PAO1 as a reference strain.(b) OD readings at 590nm of bioflim formation ability by crystal violet methods using meloxicam at sub-inhibitory conc as an anti-biofilm with PAO1 as a reference strain

the crystal violet method showed that quercetin caused a $60 \%$ reduction in biofilm formation at $93.75 \mu \mathrm{g} / \mathrm{ml}$ with P1and converting it from strong to moderate biofilm forming. While meloxicam caused

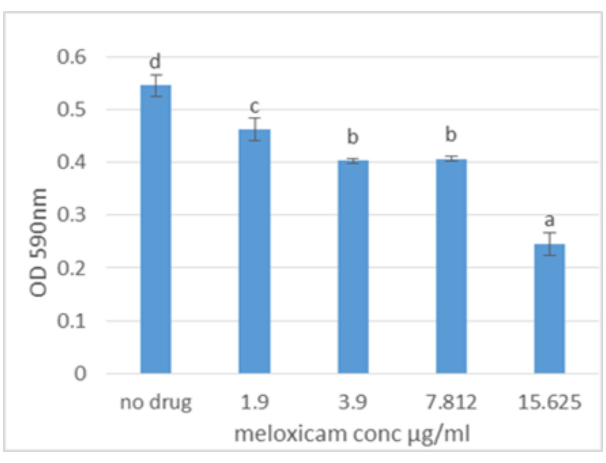

(a)
$54 \%$ inhibition at $15.62 \mu \mathrm{g} / \mathrm{ml}$ with $\mathrm{P} 1$ and converting it from strong to moderate biofilm forming. (The mean difference is significant at the 0.05 level). (Figure 11)

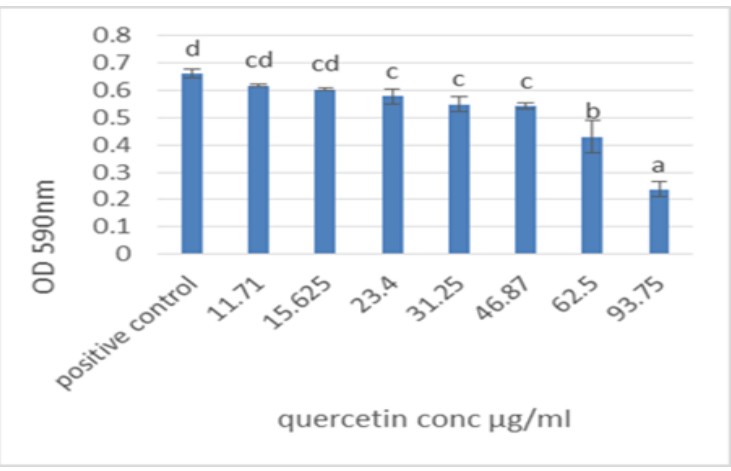

(b)

Figure (11): ( a) OD readings at 590nm of bioflim formation ability by crystal violet methods using meloxicam at sub-inhibitory conc as an anti-biofilm with $\mathrm{P} 1$ as an example of $P$. aeruginosa clinical isolate.(b) OD readings at 590nm of bioflim formation ability by crystal violet methods using quercetin at sub-inhibitory conc as an anti-biofilm with P1 as an example of $P$. aeruginosa clinical isolate.

\subsubsection{Skimmed milk agar assay for protease activity of P. aeruoginosa}

The finding showed that quercetin at $93.75 \mu \mathrm{g} / \mathrm{ml}$ caused a $62 \%$ and $61 \%$ reduction in protease production with $\mathrm{PAO} 1$ and $\mathrm{P} 1$ respectively. While meloxicam at $15.62 \mu \mathrm{g} / \mathrm{ml}$ caused $26 \%$ and
$28 \%$ reduction with $\mathrm{PAO} 1$ and $\mathrm{P} 1$ respectively. (The mean difference is significant at the 0.05 level). (Figure 12) 


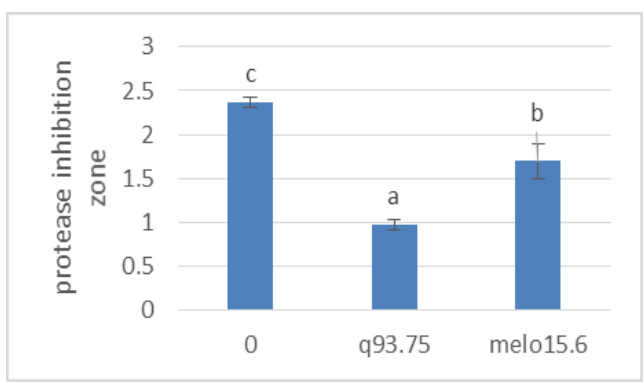

(a)

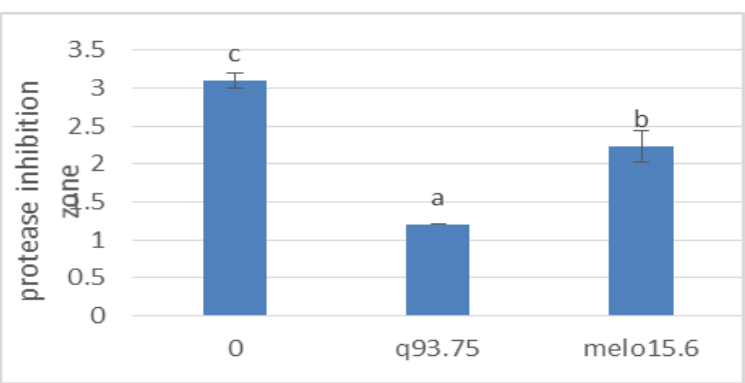

(b)

Figure 12: This figure showed the effect of both quercetin and meloxicam on protease production by measuring inhibition zone caused by this enzyme using (a) PAO1 reference strain and (b) P1 as an example of P. aeruginosa clinical isolate

\subsubsection{Rhamnolipid screening before and after treatment}

\section{Oil spreading method}

In this test the inhibitory effect of the two drugs screened by measuring inhibition zone reduction caused by the culture supernatant on the oil layer before and after treatment. PAO1 Treatment with quercetin at $93.75 \mu \mathrm{g} / \mathrm{ml}$ caused a reduction in the inhibition zone by about $74 \%$ while treating with meloxicam at $15.62 \mu \mathrm{g} / \mathrm{ml}$ causing a reduction by about $38.5 \%$. The clinical isolate P1, when treated with quercetin at $93.75 \mu \mathrm{g} / \mathrm{ml}$, caused a reduction in the inhibition zone by $55.2 \%$ while treating with meloxicam at $15.62 \mu \mathrm{g} / \mathrm{ml}$ causing a reduction by about $33.4 \%$.

(The mean difference is significant at the 0.05 level). (Figure 13)

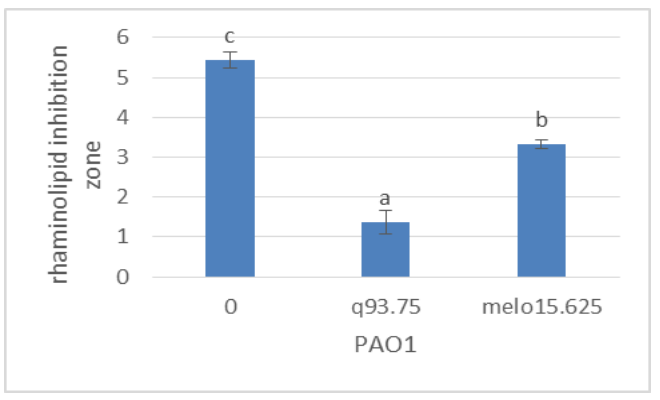

(a)

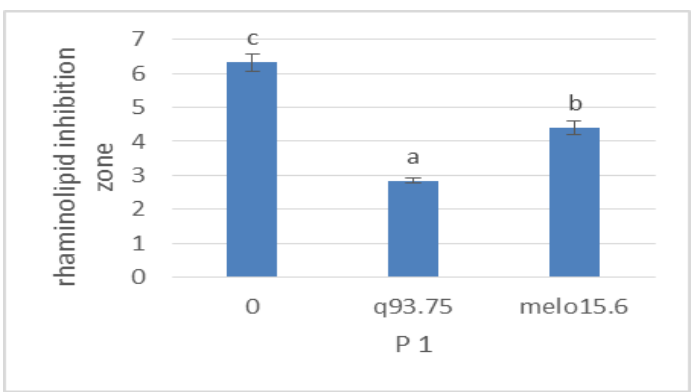

(b)

Figure 13: This figure showed the effect of both quercetin and meloxicam on rhaminolipid production by measuring inhibition zone caused by it on oil surface using (a) PAO1 reference strain and (b) P1 as an example of $P$. aeruginosa clinical isolate.

\section{Emulsification Measurement}

PAO1 was producing rhamnolipid by about $47.3 \%$ without any treatment, after culturing with quercetin at concentration $93.75 \mu \mathrm{g} / \mathrm{ml}$ it reduced to
$18.8 \%$ while after meloxicam at $15.62 \mu \mathrm{g} / \mathrm{ml}$ treatment it reduced to $28 \%$. The clinical isolate P1 ability to produced rhamnolipid was measured by 
$52.7 \%$ which is reduced to $23 \%$ by quercetin at concentration $93.75 \mu \mathrm{g} / \mathrm{ml}$ while after meloxicam at $15.62 \mu \mathrm{g} / \mathrm{ml}$ treatment reduced to $31 \%$ (The mean difference is significant at the 0.05 level). (Figure 14).

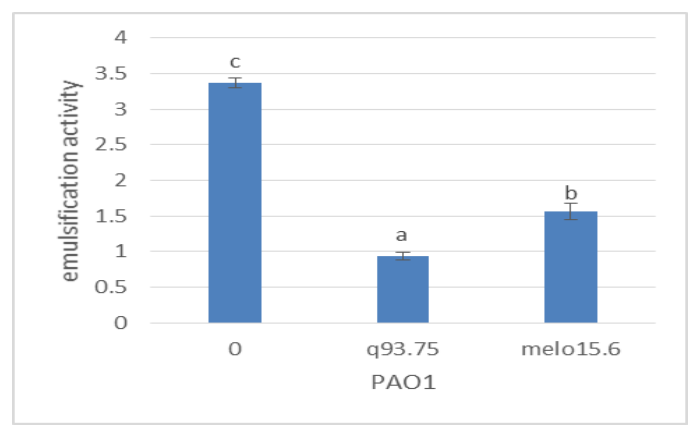

(a)

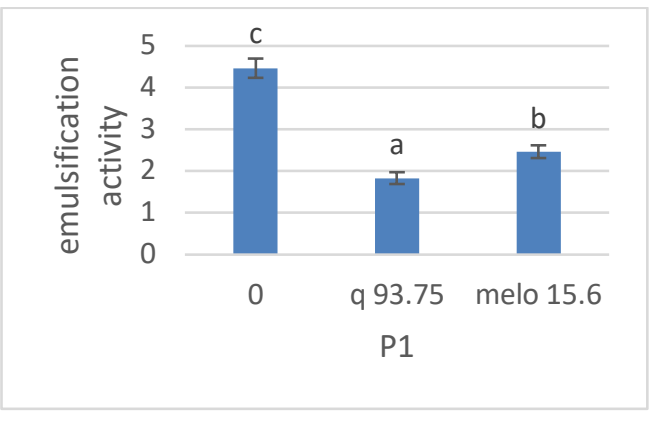

(b)

Figure (14): This figure showed the effect of both quercetin and meloxicam on rhaminolipid production by measuring the emulsification activity using (a) PAO1 reference strain and (b) P1 as an example of P.aeruginosa clinical isolate.

\subsubsection{Pyocyanin production measurement}

The results of this experiment showed that the concentration of pyocyanin produced by PAO1 \& P1 without any treatment was 1.55 and 1.48. After

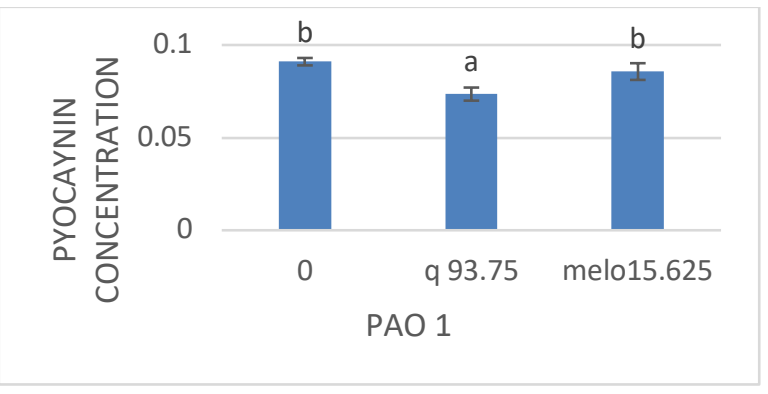

(a) culturing with quercetin at $93.75 \mu \mathrm{g} / \mathrm{ml}$ pyocyanin concentration reduced to 1.34 for both PAO1 \& P1 (The mean difference is significant at the 0.05 level). , while after treatment with meloxicam at 15.62 $\mu \mathrm{g} / \mathrm{ml}$ the pyocyanin non-significantly reduced to 1.46 for PAO1 and 1.37 for P1. (Figure 15)

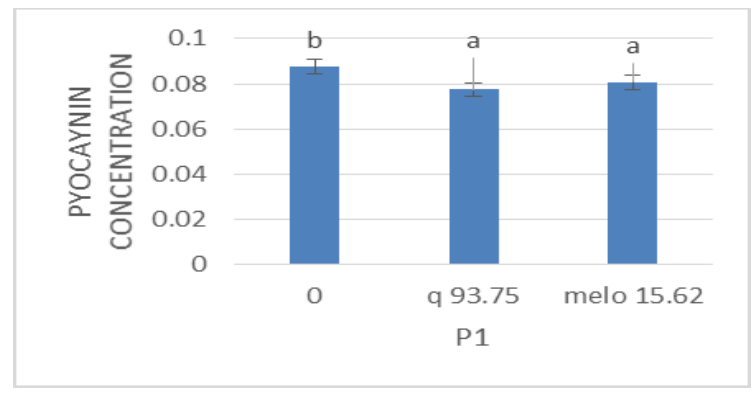

(b)

Figure 15: This figure showed the effect of both quercetin and meloxicam on pyocyanin production using (a) PAO1 reference strain and (b) $\mathrm{P} 1$ as an example of $P$. aeruginosa clinical isolate

\section{DISCUSSION}

In $P$. aeruginosa, QS modulates intercellular connection and is thanks for controlling the development of various virulence factors by tracking the population density of bacteria (via signaling molecules and receptors) ${ }^{31,32}$. There are three QS systems (lasI/R, rhlI/R, and Pseudomonas quinolone signal (PQS)) that modulate the transcription of virulence factors in $P$. aeruginosa ${ }^{33,34}$. These QS systems are the key regulators and the leading cause of the pathogenicity of $P$. aeruginosa ${ }^{34}$. Traditional antimicrobial therapy has become substantially less successful due to the rising occurrence of multi-resistant bacterial pathogens. Consequently, there is a growing need for the development of novel methods for the treatment of bacterial infections that have been the subject of modern research ${ }^{35}$. Quercetin (QCT), a natural flavonol, is abundant in all kinds of foods (e.g. tomatoes, grapes, onions, and apples) as well as in tea and wine ${ }^{36-38}$. Quercetin, as a dietary flavonoid with quorum sensing inhibitory activity ${ }^{39} \mathrm{can}$ inhibit the virulence factors and biofilm formation in P. aeruginosa ${ }^{40}$, but it has a low bactericidal activity ${ }^{41}$. Also, several studies revealed 
that certain nonsteroidal anti-inflammatory drugs (NSAIDs) possess antimicrobial activity. Chen and Wen in $2011^{42}$ reached the fact NSAIDs combined with antibiotics are effective solution against bacteria in the biofilm formation.

In our work, we studied the effect of both quercetin and meloxicam on biofilm formation and other quorum-sensing responsible virulence factors experimentally after using docking analysis as a tool of guidance to expect their efficiency.

Both quercetin and meloxicam are unlikely to be an effective antibacterial agent, but our study revealed that both have anti-biofilm activity at their sub-inhibitory concentration.

Quercetin showed a reduction in biofilm formation at all the sub-inhibitory concentrations $125-11.9 \mu \mathrm{g} / \mathrm{ml}$ by using crystal violet assay with an obvious result at $93.75 \mu \mathrm{g} / \mathrm{ml}$ as it causes about $60 \%$ reduction in biofilm formation with both PAO1 (reference strain) and also P1 (clinical isolate). In previous studies, quercetin showed a significant reduction in biofilm formation at all sub-inhibitory concentrations, but the greatest effect showed at 16 $\mu \mathrm{g} / \mathrm{ml}^{43}$ and $62.5 \mu \mathrm{g} / \mathrm{ml}^{45}$. Meloxicam also showed a significant reduction in biofilm formation with maximum effect at concentration $15.62 \mu \mathrm{g} / \mathrm{ml}$ with both PAO1 and P1. These results are following a previous publication for She et al, $2018^{46}$, which revealed that meloxicam could strongly inhibit the biofilm formation of $P$. aeruginosa and the combination treatment with antimicrobials is a promising tactic to prevent biofilm infections.

From the results of crystal violet assay for determination of biofilm formation in this study and in previous studies, quercetin and meloxicam show promise as an effective biofilm inhibitor that can be used in combinations with antibiotics. Recently, some natural products and synthetic compounds have been reported to inhibit quorum sensing virulence factors. Syzygium aromaticum extract is believed to inhibit the development of PAO1 pyocyanin $^{5}$. Curcumin, a phenolic product of Curcuma longa, also believed to attenuate PAO1 protease and elastase ${ }^{46}$. In the previous analysis, Quercetin significantly decreased $(\mathrm{P}<005)$ protease and elastase activity by 43 percent and $36 \%$ in PAO1-treated ${ }^{43}$. A further study found that a substantial reduction $(p<0.05)$ in elastase $(23$ percent) and alkaline protease (16 percent) with $15.63 \mu \mathrm{g} / \mathrm{mL}$ of meloxicam ${ }^{45}$. Pseudomonas aeruginosa proteases are considered scary virulence factors as they cause host tissue damage and interfere with antibacterial defense mechanisms of the host ${ }^{47}$. In our study, Quercetin and meloxicam demonstrated a remarkable simultaneous ability to inhibit the production of protease.

By using concentrations causing a significant reduction in biofilm formation for both drugs in determining their effect on other quorum-sensing controlled virulence factors, the data revealed that quercetin at $93.75 \mu \mathrm{g} / \mathrm{ml}$ cause $62 \%$ and $61 \%$ reduction in protease production with $\mathrm{PAO} 1$ and $\mathrm{P} 1$ respectively While meloxicam at $15.62 \mu \mathrm{g} / \mathrm{ml}$ caused $26 \%$ and $28 \%$ reduction with $\mathrm{PAO} 1$ and $\mathrm{P} 1$ respectively. (The mean difference is significant at the 0.05 level).

Quorum sensing based on $r h l$ system plays a critical role in rhamnolipid production that is needed for the incipient attachment and biofilm stability ${ }^{48}$. Rhamnolipids production showed a significant reduction in the presence of quercetin ${ }^{44}$

Soheili et al. $\quad(2015){ }^{49}$ discovered that oxicams (piroxicam and meloxicam), could well reacted with active sites of LasR by molecular and structural analysis methodology. LasR plays an important role in the initiation of the $P$. aeruginosa QS cascade system which means that it could disrupt rhamnolipid production. This finding was by our study, where quercetin and meloxicam significantly affected the rhamnolipid production which was measured phenotypically by two different methods (oil spreading method and emulsification measurement) as shown in figures 13 and 14. By disrupting rhamnolipids, proteases, and biofilm production both drugs give a promising example as quorum sensing inhibitors which can be used in combinations with antibiotics attempting to reduce antibiotic resistance and antibiotics misuse.

In this study, the effect of quercetin and meloxicam on pyocyanin production wasn't strong enough as the quercetin at a concentration of 93.75 $\mu \mathrm{g} / \mathrm{mL}$ cause around $10 \%$ reduction while meloxicam at concentration $15.62 \mu \mathrm{g} / \mathrm{mL}$ cause around $6 \%$ non-significant reduction in pyocyanin production. While in previous studies it was found that $16 \mu \mathrm{g} / \mathrm{mL}$ quercetin showed a significant negative effect on pyocyanin production ${ }^{43}$. She et al, $2017^{45}$ also found that meloxicam at concentration $15.63 \mu \mathrm{g} / \mathrm{mL}$ causes a $32 \%$ reduction in pyocyanin production. Future studies are needed to investigate the variation in quercetin and meloxicam effect on pyocyanin production by different $P$. aeruginosa strains.

\section{CONCLUSION}

$P$. aeruginosa quorum-sensing regulates virulence factors are responsible for its serious pathogenicity. Molecular docking and field alignment study are used as a tool of guidance to 
expect the activity of drugs (quercetin and meloxicam) against LasR and RhlR ligands which gave promising results to be used as quorum sensing inhibitors. Phenotypic determination of some of these QS virulence factors after treatment by the tested drugs confirmed the in silico study results. Quercetin and meloxicam can be considered as promising QS inhibitors that can be used in combination with antibiotics to enhance their effect.

\section{Conflict of interest}

The authors declare no conflict of interest.

\section{Ethics approval: NA}

Funding statement: This work was not funded

\section{Author contribution}

AGE participated in every step in this work starting from the research idea ending by writing this article. HMA participate and help mainly in handling clinical samples and microorganisms isolation and the manuscript revision before submission. DAE participated in the practical work included in this work and also revising the manuscript before submission. DHS helped in preforming all the in silico study steps (docking and field alignment technique). SMR put the main idea of the research and the methodology, followed up with every step in the practical work and revises the manuscript finally.

\section{REFERENCES}

1. Sharma G, Rao S, Bansal A, Dang S, Gupta S, Gabrani R. Pseudomonas aeruginosa biofilm: potential therapeutic targets. Biologicals. 2014;42:1-7.

2. Lyczak JB, Cannon CL, Pier GB. Lung infections associated with cystic fibrosis. Clin Microbiol Rev. 2002;15:194-222.

3. Kim C, Kim J, Park HY, Park HJ, Lee JH, Kim CK, et al. Furanone derivatives as quorumsensing antagonists of Pseudomonas aeruginosa. Appl Microbiol Biotechnol. 2008;80:37-47

4. Zeng Z, Qian L, Cao L, Tan H, Huang Y, Xue $\mathrm{X}$, et al. Virtual screening for novel quorum sensing inhibitors to eradicate biofilm formation of Pseudomonas aeruginosa. Appl Microbiol Biotechnol. 2008;79:119-26.

5. Krishnan T, Yin WF, Chan KG. Inhibition of quorum sensing-controlled virulence factor production in Pseudomonas aeruginosa PAO1 by Ayurveda spice clove (Syzygium aromaticum) bud extract. Sensors (Basel). 2012;12:4016-30.
6. Winstanley C, Fothergill JL. The role of quorum sens-ing in chronic cystic fibrosis Pseudomonas aeruginosa infections. FEMS Microbiol Lett. 2009;290:1-9.

7. Sui SJ, Lo R, Fernandes AR, Caulfield MD, Lerman JA, Xie L, et al. Raloxifene attenuates Pseudomonas aeruginosa pyocyanin pro-duction and virulence. Int $\mathrm{J}$ Antimicrob Agents. 2012;40:246-51.

8. Andrejko M, Zdybicka-Barabas A, Janczarek M, Cytrynska M. Three Pseudomonas aeruginosa strains with different protease profiles. Acta Biochim Pol. 2013;60:83-90.

9. Sarkar R, Chaudhary SK, Sharma A, Yadav KK, Nema NK, Sekhoacha M, et al. Anti-biofilm activity of Marula - a study with the standardized bark extract. J Ethnopharmacol. 2014;154:170-5.

10. Köhler T, Guanella R, Carlet J, Van Delden C. Quorum sensing-dependent virulence during Pseudomonas aeruginosa colonization and pneumonia in mechanically ventilated patients. Thorax 2010 Aug;65(8):703-10

11. Ochsner UA, Fiechter A, Reiser J. Isolation, charac terization, and expression in Escherichia coli of the Pseudomonas aeruginosa rhlAB genes encoding rhamnosyltransferase involved in rhamnolipid biosurfactant synthesis. J Biol Chem. 1994;269:19787-95.

12. Azghani AO, Miller EJ, Peterson BT. Virulence factors from Pseudomonas aeruginosa increase lung epithelial permeability. Lung. 2000;178:261-9.

13. Zulianello L, Canard C, Köhler T, Caille D, Lacroix JS, Meda P. Rhamnolipids are virulence factors that promoteearly infiltration of primary human airway epithelia by Pseudomonas aeruginosa. Infect Immun. 2006;74:3134-47.

14. Jensen PØ, Bjarnsholt $T$, Phipps $R$, Rasmussen TB, Calum H, Christoffersen L, et al. Rapid necrotic killing of polymorphonuclear leukocytes is caused by quorum-sensing-controlled production of rhamnolipid by Pseudomonas aeruginosa. Microbiology. 2007;153:1329-38.

15. Kalia VC. Quorum sensing inhibitors: an overview. Biotechnol Adv. 2013;31(2):22445.

16. Vasil ML. NA Microarrays in Analysis of Quorum sensing: strengths and Limitations. J Bacteriol. 2003;185:2061-5.

17. Nation RL, Li J. Colistin in the 21 st century. Curr Opin Infect Dis. 2009;22(6):535. 
18. Srinivas $\mathrm{P}$, Rivard K. Polymyxin resistance in gram-negative pathogens. Curr Infect Dis Rep. 2017;19:7-9.

19. Xihong Z, Yu Z, Ding T. Quorum-Sensing Regulation of Antimicrobial Resistance in Bacteria. Microorganisms. 2020 Mar;8(3):425.

20. Stephani YM, Vijayakumar G, Sunhye H, Sun C. Role of computer-aided drug design in modern drug discovery. Arch. Pharm. Res. (2015) 38:1686-1701

21. Rasmussen TB, Givskov M. Quorum sensing inhibitors: abargin of effects. Microbiology. 2006;152:895-904.

22. Stepanović S, Vuković D, Hola V, Bonaventura GD, Djukić S, Ćirković I, et al. Quantification of biofilm in microtiter plates: overview of testing conditions and practical recommendations for assessment of biofilm production by sta phylococci. APMIS. 2007;115:891-9..

23. Balaji K, Thenmozhi R, Pandian SK. Effect of subinhibitory concentrations of fluoroquinolones on biofilm production by clinical isolates of Streptococcus pyogenes. Indian J Med Res. 2013;137:963.

24. Vijayaraghavan P, Vincent SG. A simple method for the detection of protease activity on agar plates using bromocresolgreen dye. J Biochem Technol. 2013;4:628-30.

25. Youssef H, Duncan El, Nagle P, Savage N, Knapp M, McInerney J. Comparison of methods to detect biosurfactant production by diverse microorganisms. J Microbiol Methods 2004 Mar;56(3):339-47.

26. Plaza GA, Zjawiony I, Banat IM. Use the different methods for detection of thermophillic biosurfactant-Producing bacteria from hydrocarbon-contaminated and bioremediated soils. J Petrol Sci Eng. 2006;50:71-7.

27. Shamaa S Al, Bahjat S. Detection of Rhamnolipid Production in Pseudomonas aeruginosa. J Phys Conf Ser. 2019;1294(6).

28. Cooper DG, Goldenberg BG. Surface active agents from two Bacillus species. Appl Environ Microbiol. 1987;53:224-9.

29. Thenmozhi R, Nagasa A. Screening for Surface-active Agent Producing Bacteria in Used Engine Oil Contaminated Soil Samples. Res J Agric Biol Sci. 2010;6:983-6.,

30. Essar DW, Eberly L, Hadero A, Crawford I P. Identification and characterization of genes for a second anthranilate synthase in Pseudomonas aeruginosa. J. Bacteriol. 172:853-866
31. Schuster M, Greenberg EP. A network of networks: quorum-sensing gene regulation in Pseudomonas aeruginosa. Int J Med Microbiol. 2006;296:73-81.

32. Venturi V. Regulation of quorum sensing in Pseudomonas. FEMS Microbiol Rev. 2006;30:274-91.

33. Fuqua C, Greenberg EP. Signalling: listening in on bacteria: acyl-homoserine lactone signalling. Nat Rev Mol Cell Biol. 2002;3:685.

34. Smith RS, Iglewski BH. Pseudomonas aeruginosa quorum-sensing systems and virulence. Curr Opin Microbiol. 2003;6:5660.

35. Melander RJ, Melander C. The challenge of overcoming antibiotic resistance: an adjuvant approach? ACS Infect Dis. 2017;3:559-63.

36. Boots AW, Haenen GR, Bast A. Health effects of quercetin: from antioxidant to nutraceutical. Eur J Pharmacol. 2008;585:325-37.

37. Kumar A, Malik AK, Tewary DK. A new method for determination of myricetin and quercetin using solid phase microextraction-high performance liquid chromatography-ultra violet/visible system in grapes, vegetables and red wine samples. Anal Chim Acta. 2009;631:177-81.

38. Lee J, Mitchell AE. Pharmacokinetics of quercetin absorption from apples and onions in healthy humans. J Agric Food Chem. 2012;60:3874-81.

39. Vasavi HS, Arun AB, Rekha PD. Anti-quorum sensing activity of Psidium guajava L.flavonoids against Chromobacterium violaceum and Pseudomonas aeruginosa PAO1. Microbiol Immunol. 2014;58(5):286-93.

40. Paczkowski JE, Mukherjee S, McCready AR, Cong JP, Aquino CJ, Kim H, et al. Flavonoids suppress Pseudomonas aeruginosa virulence through allosteric inhibition of quorum-sensing Receptors. J Biol Chem. 2017;292(10):4064-76.

41. Sakharkar MK, Jayaraman P, Soe WM, Chow VT, Sing LC, Sakharkar KR. In vitrocombinations of antibiotics and phytochemicals against Pseudomonas aeruginosa. J Microbiol Immunol Infect. 2009;42(5):364-70.

42. Chen L, Wen YM. The role of bacterial biofilm in persistent infections and control strategies. Int J Oral Sci2011 Apr;3(2):66-73

43. Ouyang J, Sun F, Feng W, Sun Y, Qiu X, Xiong $\mathrm{L}$, et al. Quercetin is an effective inhibitor of quorum sensing, biofilm 
formation and virulence factors in Pseudomonas aeruginosa Department of Pharmacy. Southwest Hospital of the Third Military Medical University, Chongqing, China.

2016;2016: https://doi.org/10.1111/jam.1307 3.

44. Vipin C, Saptami K, Fida F, Mujeeburahiman M, Rao SS, Athmika, et al. Potential synergistic activity of quercetin with antibiotics against multidrug-resistant clinical strains of Pseudomonas aeruginosa. PLoS One. 2020 Nov 1;15(11):e0241304.

45. She P, Wang Y, Luo Z, Chen L, Tan R, Wang $\mathrm{Y}$, et al. Meloxicam inhibits biofilm formation and enhances antimicrobial agents' efficacy by Pseudomonas aeruginosa. MicrobiologyOpen.

2018;7(1): https://doi.org/10.1002/mbo3.545.

46. Rudrappa T, Bais HP. Curcumin, a known phenolic from Curcuma longa, attenuates the virulence of Pseudomonas aeruginosa PAO1 in whole plant and animal pathogenicity models. J Agric Food Chem. 2008;56:195562.

47. Ewa Ołdak and Elżbieta A. Trafny. Secretion of Proteases by Pseudomonas aeruginosa Biofilms Exposed to Ciprofloxacin. Antimicrob Agents Chemother. 2005 Aug;49(8):3281-8.

48. Bouyahya A, Dakka N, Et-Touys A, Abrini J, Bakri Y. Medicinal plant products targeting quorum sensing for combating bacterial infections. Asian Pac J Trop Med. 2017;10:729-43.

49. Soheili V, Bazzaz BS, Abdollahpour N, Hadizadeh F. Investigation of Pseudomonas aeruginosa quorum- sensing signaling system for identifying multiple inhibitors using molecular docking and structural analysis methodology. Microb Pathog. 2015;89:73-8. 